\title{
IMPLICAÇÕES ECOLÓGICAS DA FENOLOGIA REPRODUTIVA DE Salix martiana LEYB. (SALICACEAE) EM ÁREAS DE VÁRZEA DA AMAZÔNIA CENTRAL. ${ }^{1}$
}

\author{
Astrid Câmara de OLIVEIRA ${ }^{2}$, Maria Teresa Fernandez PIEDADE ${ }^{3}$
}

\begin{abstract}
RESUMO - Salix martiana Leyb. é uma planta que ocupa margens de rios de água branca, situando-se em cotas a partir de $23 \mathrm{~m}$ sobre o nivel do mar (s.n.m.). Com o objetivo de verificar a influência do tempo de inundação anual e da precipitação no comportamento fenológico desta espécie, três sitios em diferentes cotas, de 23 a 27 m (s.n.m.), foram escolhidos e em cada um deles 25 árvores foram marcadas e observadas semanalmente, durante 14 meses. Para determinação do tempo médio de formação dos frutos, três árvores por sítio tiveram 25 inflorescências marcadas, das quais $79 \%$ formaram frutos, com uma média de 48 frutos formados por inflorescência. Nào houve interferência da inundação ou da precipitação em qualquer das fenofases, sendo a produção de sementes contínua ao longo do ano. Dentro das populações não foi observada variação individual nos eventos reprodutivos. Considerando o ambiente instável habitado pela espécie, a produção ininterrupta de sementes pode representar uma importante adaptação para aumentar o sucesso reprodutivo.
\end{abstract}

Palavras-chave: floresta inundável, espécie pioneira, floração, produção de frutos.

Ecological Implications of the Reproductive Phenology of Salix martiana Leyb. (Salicaceae) in Várzea Areas of Central Amazonia.

ABSTRACT - Salix martiana Leyb, is a plant that occupies margins of whitewater rivers, at levels higher than $23 \mathrm{~m}$ above sea level (a.s.l.). In order to verify the influence of precipitation and annual inundation duration on the phenology of the species, three sites between 23 and 27 $\mathrm{m}$ a.s.l. were established. At each site, 25 individuals were labeled and observed weekly during 14 months. In order to establish the medium time for fruit formation, three individuals per site had 25 inflorescences labeled, of which $79 \%$ showed fruit formation, with 48 fruits formed per inflorescence. Neither inundation nor precipitation influenced phenology, and the production of seeds was continuous throughout the year. Considering the unstable environment the species inhabits, the pattern of seed production may be an important adaptation to increase reproductive success.

Key-words: floodplain forest, pioneer species, flowering, fruit production.

\section{Introdução}

Áreas de planície inundável ocupam na Amazônia cerca de $300.000 \mathrm{~km}^{2}$ (Junk, 1993). Em virtude de sua importância, as vegetações inundáveis têm sido descritas por diversos autores pelas suas características estruturais e floristicas (Prance, 1980; Revilla, 1981; Pires \& Prance, 1985; Klinge et al., 1995).

Nas áreas de várzea da Amazônia Central encontra-se um grande mosaico de habitats, incluindo lagos permanentes e temporários, canais e depressões pantanosas. A

\footnotetext{
'Parte da Dissertação (INPA/UA) da primeira autora.

2INPA/Max-Planck; Av. André Araújo, 2936-Petrópolis; Manaus, AM, Brasil. astrid@inpa.gov.br ${ }^{3}$ CPBA/INPA/Max-Planck; Av. André Araújo, 2936 - Petrópolis; Manaus, AM, Brasil. maitepp@inpa.gov.br
} 
vegetação inundável que coloniza estes ambientes está inserida em um gradiente de diferentes niveis de inundação, que define habitats desde permanentemente aquáticos até totalmente terrestres. Nessas áreas a amplitude de inundação varia entre 10 e $15 \mathrm{~m}$ e, o tempo de inundação pode variar de 50 a 270 dias por ano (Junk, 1989), conforme a altura de relevo considerada na planície inundável.

Salix martiana é uma espécie que coloniza áreas de várzea, formando grupamentos monoespecíficos, desde os níveis mais baixos da planície inundável, a partir da cota de $23 \mathrm{~m}$ até regiões acima da cota de $26 \mathrm{~m}$ sobre o nível do mar (Worbes, 1997). Esta espécie inicia o processo de colonização arbórea nas áreas de várzea, aproveitando-se do aparecimento de sedimentos recém expostos, crescendo rapidamente e aumentando sua densidade (Oliveira, 1998).

O gênero Salix é conhecido por sua importância econômica. Muitas de suas espécies são usadas em farmacologia, construção e movelaria, dos ramos novos de certas espécies prepara-se um tipo de vime utilizado na indústria de móveis rústicos. Portanto além da importância ecológica de $S$. martiana na sucessão dos ambientes de várzea, a espécie possui um grande potencial econômico até o momento pouco explorado na região.

Estudos envolvendo fenologia são de suma importância para a compreensão da dinâmica das comunidades florestais (Fournier \& Charpentier, 1975). Todavia, o conhecimento atual sobre fenologia é melhor desenvolvido ao nível da comunidade (Alencar et al., 1979; Augspurger, 1983; Newstrom et al., 1994). Poucos estudos enfocam populações e consideram o nível individual (Borchert, 1980; Parolin, 1996).

Muitos fatores podem estar relacionados à produção de flores e frutos, de forma que alguns autores associam os períodos de floração e frutificação a fatores abióticos. Independente do ambiente estudado, um dos fatores normalmente relacionado às fenofases reprodutivas é precipitação (Alencar et al., 1979, 1982; Ferreira, 1991). Por outro lado, existem trabalhos que levam em consideração as variações ambientais do habitat, isoladas ou acompanhadas a um ou mais fatores abióticos (Ayres, 1993; Parolin, 1996).

Nas florestas inundáveis, as flutuações anuais do nivel do rio, bem como a previsibilidade desse padrão, em virtude da sua repetição entre anos distintos (Junk et al., 1989), parecem interferir nas fenofases de diferentes espécies (Ziburski, 1991; Ayres, 1993; Wittmann \& Parolin 1999).

Este estudo teve como objetivo verificar a influência do tempo de inundação anual e da precipitação no comportamento fenológico de Salix martiana, em três populações situadas em diferentes cotas de áreas de várzea da Amazônia Central.

\section{Material e Métodos}

Este estudo foi realizado de abril de 1993 a julho de 1994. Foram escolhidos 3 sítios amostrais situados à margem esquerda do rio Solimões e 
em diferentes cotas de relevo sobre o nivel do mar (s.n.m.) com grupamentos monoespecíficos de $S$. martiana: aLira, variando entre 23 a 25 m ( $3^{\circ} 12^{\prime} \mathrm{S}$; $\left.59^{\circ} 55^{\prime} \mathrm{W}\right)$, permanecendo inundado de 6 a 8 meses; b - Cordeiro, $26 \mathrm{~m}\left(3^{\circ} 11^{\text {' }}\right.$ $\mathrm{S}$; 59 54' W), permanecendo inundado cerca de 5 meses, e c - Baixio, $27 \mathrm{~m}$ $\left(3^{\circ} 17^{\prime} \mathrm{S} ; 60^{\circ} 03^{\prime} \mathrm{W}\right)$, permanecendo inundado cerca de 4 meses. Os três sítios amostrais foram escolhidas de forma a compor um perfil do gradiente de inundação abrangendo um desnível de relevo que alcança em torno de $4 \mathrm{~m}$ (Fig. 1). Desse modo, integrando os três locais de coleta, foi possivel formar um perfil do gradiente de iundação.

Por não se dispor de dados climáticos dos referidos sítios amostrais para o período de realização deste estudo e por causa da proximidade de Manaus (Fig. 1), utilizou-se dados climáticos desse município para caracterizar a área experimental, embora sejam citadas diferenças entre o clima dessas áreas e a cidade de Manaus (Ribeiro \& Adis, 1984).

Nos sítios a e c, foram observadas 75 inflorescências de $S$. martiana, durante três semanas, 25 em cada uma de três árvores, para determinar o tempo médio de formação de frutos. $\quad \mathrm{F} \circ \mathrm{r} \mathrm{a} \mathrm{m}$ marcadas 25 árvores nos sitios a, b e c (Fig. 1), perfazendo um total de 75 indivíduos observados. As observações fenológicas foram semanais e duraram 14 meses. As fenofases estudadas foram a produção de flores e frutos. A troca de folhas não foi acompanhada porque verificou-se em observações preliminares que ela é de pequena intensidade e relativamente constante durante o ano.

A coleta de dados foi qualitativa, observando-se apenas a presença ou não de flores e frutos. Em função do tamanho da inflorescência e do tamanho do cacho de frutos, não foi possivel realizar contagem destas estruturas. Considerou-se árvores com flores aquelas com amentilhos visiveis e com frutos as árvores nas quais foi possivel visualizar cachos com capsulas fechadas, ou seja, que ainda abrigavam as sementes.

\section{Resultados}

Acompanhando as inflorescências foi possivel verificar que a formação de flores assim como de frutos ocorre da região basal para a região apical do amentilho, em um processo cuja duração, considerando a inflorescência como um todo, é em torno de três semanas. Entretanto, cada flor leva cerca de quatro dias para formar um fruto. Após cinco dias, todas as inflorescências marcadas já continham frutos. Das 25 inflorescências marcadas em cada uma das três árvores, um total de $79 \%$ formaram frutos: 16 na árvore 1, 24 na árvore 2 e 19 na árvore 3. A média de frutos formados por inflorescência marcada foi de 45,4 frutos na árvore $1,52,3$ frutos na árvore 2 e 47,2 frutos na árvore 3.

Durante todo o período de 14 meses de observações, pelo menos $84 \%$ das plantas acompanhadas se encontravam florescendo e frutificando ininterruptamente, 

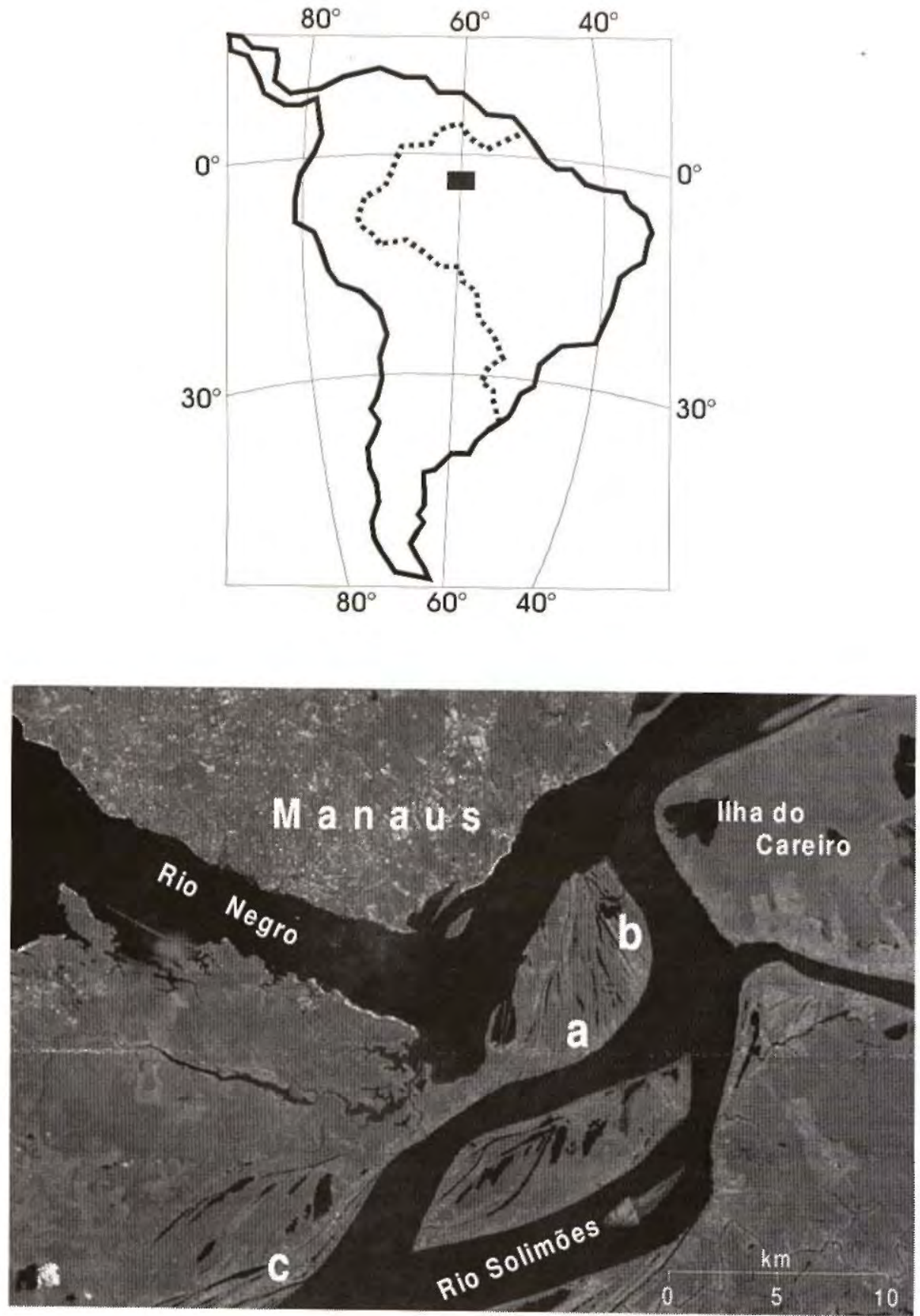

Figura 1. Mapa da área de estudo, no baixo rio Solimões, indicando os três sítios amostrais: a) Lira, 23 a 25 m sobre o nível do mar (s.n.m.), b) Cordeiro, 26 m s.n.m., c) Baixio, 27 m s.n.m. 
independente do sítio amostral considerado. Uma vez que as fenofases floração e frutificação sạo subsequentes e com curtos intervalos, a Fig. 2 refere-se a ambas as fenofases. O mesmo padrão foi observado, independente do diferente período de inundação ao qual cada uma das populações estudadas foi submetida, e independente do tamanho das plantas consideradas. Tampouco foi observada variação individual dentro da população.

\section{Discussão}

O padrão de floração individual é definido pela duração da floração, bem como pelo número e a distribuição temporal de flores
(Augspurger, 1983). Gentry (1974) afirmou existirem espécies tropicais em um extremo, onde indivíduos produzem grande número de flores novas a cada dia durante uma semana ou menos, e em outro, onde indivíduos produzem pequeno número de flores novas quase diariamente durante muitas semanas. Em $S$. martiana tal padrão não ocorre: esta espécie produziu grande quantidade de flores e frutos durante todo o período de 14 meses de estudo.

Acompanhando 29 espécies de igapó, Ferreira (1991) observou que $27 \%$ tem seu periodo de floração e $55 \%$ o periodo de frutificação significativamente relacionados com a variação média do nível do rio; $59 \%$
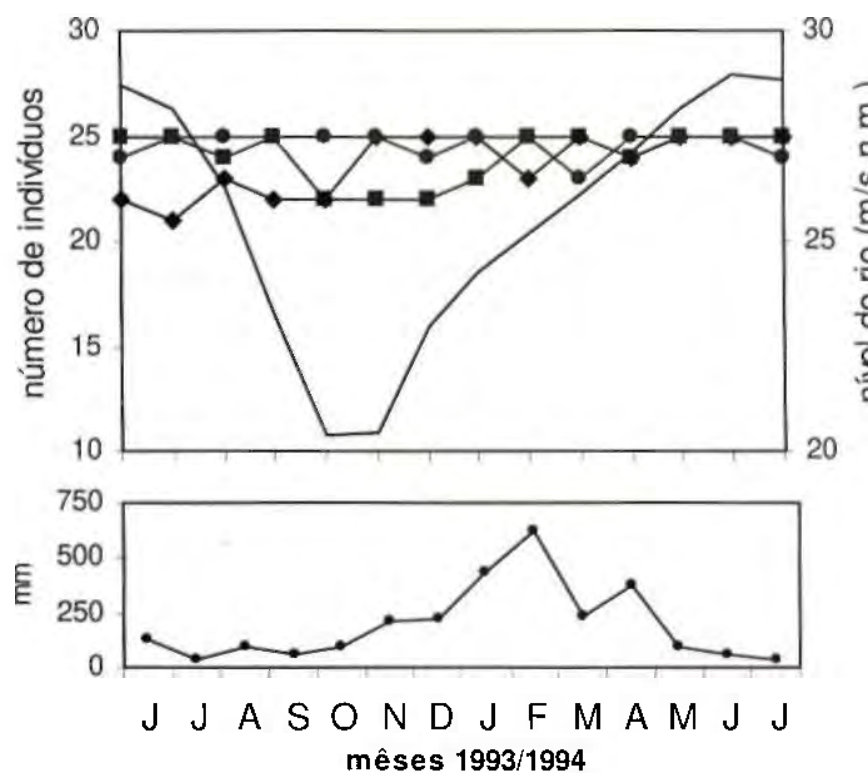

Figura 2. Númcro de individuos de Salix martiana produzindo flores e frutos em relação à flutuação do nivel do rio (s.n.m.), no periodo de 1993/1994, nos sitios a) 23 a 25 m s.n.m., b) $26 \mathrm{~m} \mathrm{s.n.m.} \mathrm{e} \mathrm{c)} 27 \mathrm{~m}$ s.n.m. 
tem sua floração e $55 \%$ a frutificação significativamente correlacionados com a variação média da precipitação. Diferentemente, a espécie deste estudo não parou de produzir flores e frutos ao longo de todo o período amostral, sugerindo que sua fenologia reprodutiva independe das flutuações do nivel do rio e da precipitação.

Em áreas de várzea da região do Mamirauá, Ayres (1993) relacionou períodos de floração e frutificação à flutuação do nível do rio, como forma de favorecer mecanismos específicos de dispersão. Espécies hidrocóricas e ictiocóricas, como também aquelas dispersadas por outros animais, tendem a apresentar o pico de maturação e queda dos frutos no pico da fase aquática. Particularmente a dispersão hidrocórica pode ser considerada mais adaptativa nesses ambientes, e mais eficiente do que aquela feita por outros agentes dispersores, pois permite que as sementes que flutuam, sendo levadas para locais mais distantes, possam apresentar uma distribuição mais eqüitativa e colonizar novos ambientes. Já as plantas cujos frutos têm síndromes anemocóricas, como Pseudobombax munguba (Bombacaceae), dispersam suas sementes quando o nível da água está começando a descer (Ayres, 1993; Ziburski, 1991). Embora S. martiana seja uma espécie com dispersão anemocórica (Cronquist, 1981; Kubitzki, 1985) e também hidrocórica, uma vez que suas sementes flutuam, os padrões de produção de frutos da espécie não correspondem àqueles encontrados para essas síndromes de dispersão por Ayres (1993) e Ziburski (1991).
Augspurger (1981), em estudo sobre a sincronia reprodutiva de Hybanthus prunifolius (Violaçeae), observou que populações nas quais há sincronia nos eventos reprodutivos em condições naturais, houve produção bem maior de sementes, do que em individuos onde foi induzida a assincronia na população. Em áreas de igapó da Amazônia brasileira, Ferreira et al. (no prelo) encontraram clara variação intraespecifica na fenologia reprodutiva de Eschweilera parvifolia (Lecythidaceae) em relação a posição das plantas ao longo do gradiente de inundação. Entretanto, nas populações de $S$. martiana observadas durante este estudo, pode-se sugerir que, independente da cota onde as plantas estão situadas, ocorre forte sincronia dos eventos reprodutivos dentro da população. Sendo uma espécie anemófila, que habita um ambiente difícil de ser colonizado, a produção ininterrupta de flores e frutos pode representar um ganho, especialmente pelo fato da espécie ser autocompatível (Rohwer \& Kubitzki, 1984), podendo cada individuo produzir seus próprios frutos, além de poder sofrer também fecundação cruzada.

Parolin (1996), estudando duas espécies pioneiras da várzea, Cecropia latiloba (Cecropiaceae) e Senna reticulata (Caesalpiniaceae), observou que, para essas espécies, o pico de frutificação ocorreu no final da vazante. Trabalhando com Vitex cymosa (Verbenaceae) e Cecropia latiloba, Ziburski (1991) observou padrão igual. Neste estudo, o autor 
também relaciona o pico de frutificação com a sindrome de dispersão, que no caso de $V$. cymosa é barocórica. S. martiana é também uma espécie pioneira e não demonstrou padrões semelhantes aos das demais espécies estudadas, embora todas colonizem o mesmo ambiente.

Ao contrário das espécies descritas anteriormente, tanto em função de sua síndrome de dispersão, quanto em função da variação do nível do rio e precipitação, a produção de flores e frutos em $S$. martiana foi contínua e independente da posição ocupada pelas plantas no gradiente de inundação. Dessa forma, seu padrão de floração e frutificação pode ser enquadrado na classificação feita por Newstrom et al. (1994), como uma freqüência de floração contínua, com curtas e raras interrupções, as quais não foram observadas para $S$. martiana. Embora para a várzea amazônica apenas Crescentia amazonica (Bignoniaceae) seja citada por Kubitzki \& Ziburski (1994) como espécie que produz frutos continuamente ao longo do ano todo, esse fenômeno é referido como comum em muitos grupos de plantas de sucessão secundária em terra-firme, podendo ser verificados exemplos em várias espécies das famílias Piperaceae, Malvaceae, Melastomataceae e Asteraceae (Gómez-Pompa \& Vázquez-Yanes, 1974; Vázquez-Yanes \& OrozcoSegovia, 1992).

Outro aspecto que ainda pode ser observado é que provavelmente o comportamento fenológico de $S$. martiana esteja associado à sua condição ecológica de estrategista " $r$ " (Pianka, 1970; 1994). Esses etrategistas apresentam altas taxas de reprodução e colonizam ambientes normalmente desfavoráveis a muitas formas de vida, onde ocorrem desgastes mecânicos (Remmert, 1982). As árvores de $S$. martiana situadas nas cotas mais baixas (entre 23 e $25 \mathrm{~m}$ ), podem permanecer inundadas entre 8 e 9 meses, como ocorreu no período deste estudo.

Considerando que às margens do rio Solimões existe uma forte correnteza, o ambiente colonizado por S. martiana propicia uma série de abalos mecânicos, seja pela erosão ou pela água, ou por restos de vegetais flutuando, como troncos e grandes massas de capins. Por outro lado, essas plantas possuem pouco tempo para germinar e crescer antes da subida das águas (Oliveira, 1998). A produção continua de grande quantidade de sementes provavelmente é uma adaptação para aumentar o sucesso reprodutivo de Salix martiana, em um ambiente instável, com vários eventos catastróficos, que freqüentemente eliminam grandes quantidades de plântulas já nas primeiras semanas do estabelecimento (Oliveira, 1998).

\section{Agradecimentos}

Este estudo foi possível graças ao financiamento do projeto INPA/MaxPlanck e programa SHIFT de cooperação Brasil/Alemanha. A CAPES forneceu uma bolsa de estudos a primeira autora. Agradecemos a Valdeney Azevedo e Auristela 
Conserva pela ajuda no trabalho de campo, bem como ao pessoal de apoio do BADPI.

\section{Bibliografia citada}

Alencar, J. da C.; Almeida, R.A. de; Fernandes, N.P. 1979. Fenologia de espécies florestais em floresta tropical úmida de terra firme na Amazônia Central. Acta Amazonica, 9(1):163-198.

Augspurger, C.K. 1981. Reproductive synchrony of tropical plants: experimental effects of pollinators and seed predators on Hybanthus prunifolius (Violaceae). Ecology, 62:775-788.

Augspurger, C,K, 1983. Phenology, flowering synchrony, and fruit set of six neotropical shrubs. Biotropica, 15(4):257-267.

Ayres, J.M. 1993. As matas de várzea do Mamirauá. médio rio Solimões. $\mathrm{CNPq}$ Programa Trópico Úmido e Sociedade Civil Mamirauá, Brasilia, DF. 123p.

Borchert, R. 1980. Phenology and ecophysiology of tropical trees: Erythrina poeppigiana O. F. Cook. Ecology, 61(5):1065-1074.

Cronquist, A. 1981. An integrated system of classification of flowering plants. Columbia University Press, New York. 1262p.

Ferreira, L.V. 1991. O efeito do periodo de inundação na zonação de comunidades. Fenologia e regeneraçẫo em uma floresta de igapó na Amazônia Central. Dissertação de Mestrado, INPA - FUA, Manaus, AM, 161p.

Fournier, L.A.; Charpentier, C. 1975. El tamaño de la muestra y la frecuencia de las observaciones en el estudio de las características fenológicas de los árboles tropicales. Turrialba, 25(1):45-48.

Gentry, A.H. 1974. Flowering phenology and diversity in tropical Bignoniacae. Biotropica, 6:64-68.

Gomes-Pompa, A.; Vázquez-Yanes, C. 1974. Studies on the secondary sucession of tropical lowlands: life cycle of secondary species. Proceedings of 1 st International Congress of Ecology, W. Junk Publishers, the Hague. pp 336-342.

Junk, W.J. 1989. Flood tolerance and tree distribution in Central Amazonian floodplains. In: Nielsen L.B., Nielsen I.C., Balslev H. (Eds.) Tropical forest: botanical dynamics, speciation and diversity. Academic Press, London. pp 47-64.

Junk, W.J.; Bayley, P.B.; Sparks, R.E. 1989. The floodpulse concept in the river-

floodplain systems. Canadian Special Publication in Fisheries and Aquatic Sciences, 106:110-127.

Klinge, H.; Adis, J.; Worbes, M. 1995. The vegetation of a seasonal várzea forest in the lower Solimões River, Brazilian Amazonia. Acta Amazonica, 25(3/4):201-220.

Newstrom, L.E.; Frankie, G.W.; Baker, H.G. 1994. A new classification for plant phenology based on flowering patterns in lowland tropical rain forest trees at $\mathrm{La}$ Selva, Costa Rica. Biotropica, 26:141-159

Oliveira, A.C. 1998. Aspectos da dinâmica populacional de Salix martiana Leyb. (Salicaceae), em áreas de várzea da Amazonia Central. Dissertação de Mestrado, INPA - FUA, Manaus, AM. 83 p.

Parolin, P. 1996. Auswirkungen periodischer Vernässung und Überflutung auf die Phänologie, Photosynthese und Blattphysiologie von Baumarten unterschiedlicher Wachstumsstrategien in $z$ e n tral a mazonischen Überschwemmungsgebieten. Doktor Arbeit, Universität Hamburg, Hamburg. 156p.

Pianka, E.R. 1970. On r - and K - selection. Am. Nat., 104:592-597.

Pianka, E.R. 1994. Evolutionary Ecology. 5th ed. The University of Texas at Austin, Austin, 486p.

Pires, J.M.; Prance, G.T. 1985. The vegetation types of the Brazilian Amazon. In: Prance G.T.; Lovejoy T.E. (Eds). Amazonia-Key Enviroments. Pergamon Press, London. pp 109-145.

Prance, G.T. 1980. A terminologia dos tipos de florestas amazônicas sujeitas à inundação. Acta Amazonica, 10(3):495-504. 
Remmert, H. 1982. Ecologia. Edusp, São Paulo. 335p.

Ribeiro, M.N.G.; Adis, J. 1984. Local rainfall variability - a potential bias for bioecological studies in the central Amazon. Acta Amazonica, 14(1/2): 159-174.

Rohwer, J.; Kubitzki, K. 1984. Salix martiana, a regularly hermaphrodite willow. Pl. Syst. Evol., 144:99-101.

Vázquez-Yanes, C.; Orozco-Segovia, A. 1992. Effects of litter from a tropical rainforest on tree seed germination and establishment under controlled conditions. Tree Physiology, 11:391-400.

Wittmann, F.; Parolin, P. 1999. Phenology of six tree species from Central Amazonia várzea. Ecotropica, 5:51-57.

Ziburski, A. 1991. Dissemination, Keimung und Etablierung einiger Baumarten der Überschwemmungswälder Amazoniens. Tropische und Subtropische Pflanzenwel. 77:1-96.

Aceito para publicação em 03/06/2002. 